\title{
Stage de récupération de points du permis de conduire : Quelles activités des formateurs?
}

Training course allowing points back of driving licence in France: What trainers real activities?

\section{Alain Jean}

\section{(2) OpenEdition}

\section{Journals}

Édition électronique

URL : http://journals.openedition.org/activites/2724

DOI : 10.4000/activites. 2724

ISSN : 1765-2723

Éditeur

ARPACT - Association Recherches et Pratiques sur les ACTivités

\section{Référence électronique}

Alain Jean, « Stage de récupération de points du permis de conduire : Quelles activités des

formateurs ? ", Activités [En ligne], 13-1 | 2016, mis en ligne le 15 avril 2016, consulté le 20 avril 2019.

URL : http://journals.openedition.org/activites/2724 ; DOI : 10.4000/activites.2724

Ce document a été généré automatiquement le 20 avril 2019.

\section{(i) $\ominus$}

Activités est mis à disposition selon les termes de la licence Creative Commons Attribution - Pas d'Utilisation Commerciale - Pas de Modification 4.0 International. 


\section{Stage de récupération de points $d u$ permis de conduire: Quelles activités des formateurs?}

Training course allowing points back of driving licence in France: What trainers real activities?

Alain Jean

\section{NOTE DE L'ÉDITEUR}

Article soumis le 9 mars 2015, accepté le 19 octobre 2015.

\section{Introduction}

1 La loi $n^{\circ}$ 89-469 du 10 juillet 1989 a instauré le système du permis à points en France. Ce système qui n'est pas une spécificité française vise à responsabiliser les conducteurs qui doivent participer à la baisse de la mortalité routière en changeant leurs comportements sur la route. Il s'inscrit également dans une démarche pédagogique dont le but est d'éviter l'infraction et sa récidive. Plusieurs possibilités existent pour récupérer les points perdus et reconstituer son capital : délai de six mois, délai de trois ans et... suivre un stage de sensibilisation à la sécurité routière.

2 La formation est dispensée dans toute la France, par l'intermédiaire d'associations, à des groupes constitués d'environ 20-25 stagiaires. Les sessions sont assurées par deux animateurs formant un binôme sur les deux jours et rémunérées pour cela. Le binôme est constitué par l'association qui choisit le lieu, programme l'intervention et procède aux inscriptions des conducteurs infractionnistes. Les textes prévoient notamment des études de cas d'accidents, un exposé sur les lois physiques ainsi que leurs conséquences sur les véhicules et leur conduite. Le stage, d'une durée de deux jours, se termine par un 
questionnaire d'auto-évaluation. Une attestation est remise au conducteur qui a suivi la totalité de la formation. Un double de ce document est transmis au préfet du département du lieu de suivi de stage dans un délai de 15 jours. Il est alors procédé à la restitution de quatre points qui prend effet le lendemain de la dernière journée du stage. Le coût du stage varie de 230 à $280 €$ selon les associations.

Plus concrètement, une majorité des personnes qui s'inscrivent à ces stages ont pratiquement perdu leur capital de points. En effet, un conducteur n'ayant plus de points n'a plus le droit de conduire si une infraction lui en retire encore. Il faut donc ne jamais passer en dessous de zéro point, si on ne veut pas retenter toute la procédure de passage d'un permis de conduire. Les personnes venant assister à un stage sous peine de se trouver dans l'impossibilité de se déplacer en voiture de manière autonome estiment la plupart du temps être victimes d'injustices et que les mauvais conducteurs sont toujours les autres. L'intention que l'on repère chez certains stagiaires est de régler des comptes avec les formateurs qu'ils pensent être les représentants du système responsable de l'injustice dont ils ont été victimes. De plus les deux jours de stage étant consécutifs, les stagiaires doivent prendre sur leurs congés pour le suivre. Bref, l'ambiance est souvent agressive, les conflits nombreux dégénérant parfois en rixes comme nous allons le voir plus loin. La formation des formateurs est abordée de manière institutionnelle : elle porte sur les prescriptions des stages, des principes de méthodes d'apprentissage. Il n'en demeure pas moins que les formateurs une fois sur le terrain rencontrent de grosses difficultés, et force est de constater qu'ils « dévient » fortement des prescriptions, malgré des visites d'inspecteurs vérifiant la conformité des stages. Les formateurs estiment que l'application stricte des contenus prescrits génère des insatisfactions des stagiaires, accentuant leur agressivité. Entre prescriptions, efficacité estimée, implication des stagiaires et intérêts pédagogiques, les formateurs semblent obligés de s'ajuster, de procéder à des arrangements (Derouet, 1988), des genèses instrumentales (Rabardel, 2002) sous peine de voir les deux jours de stage prendre des allures de guerre de tranchées entre formateurs et stagiaires, mais également quelquefois entre formateurs eux-mêmes. La diversité des publics, en termes de statuts sociaux, d'obligations et de démarches pratiquement imposées, joue aussi un rôle important dans l'activité déployée par les formateurs dans ce type de stage.

4 Nous avons mené une recherche sur l'activité réelle mobilisée par les formateurs durant un stage, en regard des prescriptions nationales arrêtées par la Sécurité routière ${ }^{1}$. Nous avons voulu comprendre la dynamique de ce type de stage du point de vue des formateurs et la mettre en relation avec les prescriptions. Plus précisément, dans le cadre de la didactique professionnelle (Pastré, 2011), nous nous sommes interrogés sur les genèses opératives, et les concepts pragmatiques, mobilisés par les animateurs de ces stages lorsqu'ils s'ajustent aux situations souvent problématiques qu'ils rencontrent. Cette recherche n'émanait pas d'une commande précise, mais d'un constat de l'association concernée d'un turn-over important des formateurs dans ce type de stage. La coanimation de deux formateurs de statut différents étant une particularité de ces formations, l'association voulait essayer de comprendre ce qui pouvait se jouer en tant qu'ajustements respectifs dans un binôme qui fonctionnait ainsi depuis quelque temps. 


\section{Bref historique des stages de sensibilisation à la sécurité routière}

5 Votée en 1989, la loi instaurant le permis à points est devenue applicable en juillet 1992. Définissant le champ d'action, l'arrêté d'application précise également les moyens mis en œuvre pour l'animation des stages de sensibilisation aux causes et conséquences des accidents de la route. Depuis leur création, le législateur a voulu que ces stages soient animés par deux formateurs de statut différent. Ainsi le binôme est obligatoirement constitué d'un formateur titulaire d'un BAFM (Brevet d'Aptitude à la Formation des Moniteurs d'enseignement à la conduite des véhicules terrestres à moteur) et d'un psychologue diplômé d'un master.

6 L'instauration du système permis à points peut se décliner en plusieurs finalités. Sur le plan de la santé publique, la baisse de la sinistralité est l'objectif général recherché. Un autre objectif est de considérer le permis à point comme un outil de responsabilisation des conducteurs par le retrait de points et par la traçabilité de(s) l'infraction(s). La formation continue du conducteur étant inexistante, la participation volontaire ou obligatoire au stage de sensibilisation permettrait de pallier ce manque. Cette organisation permet une sélection a priori des conducteurs tels que les multirécidivistes de l'infraction ou, dès 2004, les conducteurs novices pour leur forte sinistralité.

7 Le programme de cette formation est décrit par l'annexe II de l'arrêté du 26 juin 1992. Les formateurs, eux, suivent une formation spécifique de cinq semaines pour pouvoir animer des stages.

8 Le module dit stage de $2^{\text {ème }}$ génération se détermine par les termes suivants: " réhabilitation des conducteurs infractionnistes ». Il présuppose donc un préalable de dysfonctionnement physique ou normatif. Ainsi, «Le mode d'intervention choisi vise à impulser un processus de changement d'attitudes et de comportement chez le conducteur en cohérence avec l'objectif des programmes de réhabilitation pour conducteurs infractionnistes (modifier les comportements pour prévenir la réitération d'infractions, ce qui entraînera une amélioration de la sécurité). Les deux modèles théoriques retenus pour la création de ces modules appartiennent pour le premier au modèle hiérarchique du conducteur (Keskinen, 1996) et pour le second à Prochaska, Di Clemente et Norcross (1992, psychologie théorique de santé avec l'autodiagnostic et l'éducation comme ancrage d'une démarche psychologique éducative)

\section{Objets de notre recherche}

9 Les textes officiels (article R223-5 du Code de la Route), prescrivent les conditions de déroulement de ces stages et en précisent les objectifs.

On ne repère qu'assez peu de flexibilité, dans le cahier des charges que nous nous sommes procuré, et dans les discours des acteurs qui le perçoivent comme très étroit et très prescrit. Tout laisse à penser que, par rapport aux deux types de flexibilité proposés par Béguin et Cerf (2004, p. 59) susceptibles d'être intégrés dans la conception d'artefacts, nous sommes plutôt dans une faible plasticité synchronique, les concepteurs du stage s'imaginant prescrire et prévoir à l'avance l'activité des opérateurs. Toutes les visites sur 
les sites web des associations agréées par les préfectures, proposant des stages de tronc commun, déclinent ces prescriptions selon un même déroulé :

11 Premier jour

Accueil des participants

Tour de table

Chiffres de la Sécurité routière

Traitement de l'infraction

Système de permis à points

Notions de physiologie. (Le temps de réaction, la vue, la vigilance, la fatigue...)

\section{Deuxième jour}

Étude de cas d'accident

Accidentologie

Lois physiques et limites des véhicules

Alcool et conduite

Bilan du stage

Nous nous sommes interrogés sur les activités déployées par les formateurs lors de ce type de stage pour s'ajuster au public et aux situations. Plus précisément, nous avons voulu rechercher :

- Les genèses instrumentales (Rabardel, 1995) opérées par les formateurs vis-à-vis du dispositif de formation de récupération de points considéré comme un artefact

- Les concepts pragmatiques (Pastré, 2011) mobilisés par les formateurs.

- Les combinaisons de gestes professionnels d'ajustement (Bucheton, 2009) mobilisées par les formateurs pour s'ajuster aux imprévus lors de ce stage.

Les présupposés qui étaient les nôtres dans cette étude portaient sur le concept d'artefact qui désigne "de façon neutre toute chose finalisée d'origine humaine. Les artefacts peuvent aussi bien être matériels que symboliques. Un artefact peut avoir différents statuts pour le sujet et notamment, celui qui nous intéresse ici, le statut d'instrument lorsqu'il est moyen de l'action pour le sujet » (Rabardel, 2002). Ainsi nous considérons que le dispositif de formation du type "sensibilisation à la conduite routière " peut se concevoir comme un artefact lié à un système de travail présenté sous la forme de prescriptions avec son cahier des charges et ses supports de formation. Le système de travail étant alors celui conçu pour :

1. être décliné en programme de formation par des associations très diverses.

2. être mis en œuvre, à partir de ce programme de formation, sur tout le territoire national par des binômes de formateurs, sélectionnés et rémunérés par ces associations.

Compte tenu de ce qui précède, de ce type de prescriptions, du grand nombre de stages à assurer et des constitutions aléatoires des binômes, nous avons émis l'hypothèse qu'il était nécessaire pour les formateurs d'effectuer des conceptions dans l'usage à partir des prescriptions nationales peu flexibles. Plus exactement que les binômes effectuaient des conceptions alternées dans l'usage, pour pouvoir assurer ces formations.

\section{Méthodologie de recueil de données}

Les entretiens semi-directifs que nous avons menés avec 5 formateurs ( 3 formateurs titulaires d'un BAFM et 2 psychologues) ont montré qu'une bonne partie des stages ne suivait pas le déroulé affiché par les associations qui les emploient. Les stages ne suivent que partiellement les prescriptions nationales, car certaines «ne passent pas auprès de 
stagiaires ». Le stage que nous avons suivi était assuré par deux formateurs ayant l'habitude de travailler ensemble au hasard des affectations d'une association très présente dans notre département, qui assure environ $30 \%$ des formations. Bien entendu, les formateurs étaient d'accord sur la méthode de la recherche, notamment sur le principe d'autoconfrontations à partir de traces de leur activité en situation de stage. La situation de formation, dont nous proposons de présenter et mettre en discussion l'analyse que nous avons faite dans notre recherche, correspondait à ce que nous cherchions : elle a été qualifiée par les formateurs, comme un stage de routine, constitué d'un public plutôt classique, avec des postures quelquefois agressives, mais ne se situait pas dans ce qu'ils avaient connu de plus conflictuel.

Le stage s'est déroulé en janvier 2013. Nous nous sommes mêlés aux stagiaires et avons participé au déroulé.

18 Seize stagiaires étaient présents dont la diversité est ainsi répartie : 2 commerciaux, 1 employée de restauration, 1 chauffeur routier, 1 architecte, 2 élagueurs, 1 manufacturier d'entreprise, 1 paysagiste, 1 électricien bâtiment, 1 organisateur événementiel, 1 universitaire, 1 gestionnaire, 1 étudiant, 1 infirmière, 1 agent hospitalier. Sur ces seize stagiaires, trois femmes seulement, cinq jeunes de moins de 22 ans, onze personnes entre 22 et 40 ans, cinq personnes de plus de 40 ans.

19 Le binôme formateur était constitué de Valentin le psychologue et de Noémie la formatrice BAFM.

20 Nous avons procédé à des recueils de traces de l'activité des formateurs pendant le stage sous forme d'enregistrements audio. Pour des raisons de disponibilités des formateurs, nous n'avons pas réussi à les réunir à nouveau. Nous avons donc procédé séparément avec chacun à un entretien d'autoconfrontation simple à partir de ces traces. Il n'était pas envisageable pour eux d'écouter les 12 heures d'enregistrement. Nous l'avons donc fait en préalable en croisant avec les notes prises pendant le stage, afin de repérer ce que nous estimions être des imprévus. L'entretien consistait d'une part à confirmer que ces imprévus perçus par nous l'étaient également pour le formateur, et d'autre part à renseigner les préoccupations, les raisons qui guidaient leurs traitements. Nous avons également demandé à chacun, s'il désirait s'exprimer sur un moment particulier du stage et procédé à l'écoute de ces sélections. Les entretiens ont ainsi duré entre deux heures et deux heures trente. Bon nombre d'imprévus ont été confirmés séparément par chacun des formateurs.

$21 \quad 1^{\circ}$ jour

8h45 Présentation de l'association organisatrice par son président

$\mathbf{8 h 5 0}$ Valentin (Psychologue) présente le stage. Les comportements, l'alcoolémie et les symptômes, nos petits défauts, toxicomanie

9h20 Noémie : Les horaires du stage, la feuille d'émargement.

Les différents types de stagiaires, historique du code de la route

«Alors très rapidement, on fait un tour de table!»

Valentin prend la parole «Vous vous présentez rapidement et répondez à trois questions: quel est le motif de votre venue ici? Quel type de véhicule conduisez-vous? Avez-vous déjà eu un accident générant un blessé? "...

... Le tour de table se terminera le soir à $17 \mathrm{~h} . .$.

$222^{\circ}$ jour

$\mathbf{8 h 0 0}$ Préparation des attestations de stage. Questions

$\mathbf{8 h 0 5}$ Présentation de chiffres sur les accidents de la route. Vitesse et ressenti. Les situations à risque 
$14 \mathrm{~h}$ Les temps de réaction

$15 \mathrm{~h}$ Perceptions et images ambiguës. Distances de freinage et temps de réaction.

Projection d'un spot d'un choc

$17 \mathbf{h}$ Fin du stage et remise des attestations de stage our de table qui débute à 9 h20 et se termine à $17 \mathrm{~h}$, se déroule quasiment sur la même structure à partir de la rapide présentation de chacun des participants et de leurs réponses aux trois questions posées. Valentin prend la parole et développe l'infraction du stagiaire à l'aide des risques associés, des raisons de la verbalisation et des peines encourues. Ce développement prend plus ou moins de temps en fonction des exemples qu'il prend, de son expérience sur le sujet et de l'actualité des accidents dans la région. Pour les premières présentations, il aborde un historique du code de la route qui est complété de temps en temps par Noémie. Il aborde souvent les risques de l'utilisation du téléphone portable en conduisant, en exposant des solutions adoptées par certaines entreprises européennes, mais étrangères à la France. Il pose des questions de connaissance au stagiaire, puis au groupe sur les thèmes qu'il aborde. Pendant cette phase, le stagiaire concerné tente quelquefois de minimiser son infraction ou de rejeter la faute qu'il a commise sur d'autres usagers ou des catégories d'usagers (les hommes, les femmes au volant, " les jeunes », « les vieux »...). Une deuxième phase consiste à prendre des exemples de signalisations routières relatives à l'infraction et aux différents véhicules dans des zones proches du lieu du stage et à s'assurer que la majorité des stagiaires en aient une représentation précise. Il s'agit pour lui de faire comprendre la logique de présence ou d'absence de ces signalisations selon des stratégies de sécurité et non de verbalisation. C'est une phase où les stagiaires interviennent le plus souvent pour contester son discours. La dernière phase consiste à relancer lorsque le stagiaire a déjà eu " un accident grave générant un blessé ». Trois ou quatre stagiaires étaient dans ce cas, ce qui lui a permis de démontrer la gravité du non-respect des signalisations ou des règles du code de la route. Pendant ce développement Valentin aborde régulièrement les thèmes suivants : l'homme et son rapport à la loi, la quête du bonheur, la valorisation de l'homme au travers d'une voiture, de sa puissance ou de sa vitesse...

25 Pendant le tour de table, Noémie intervient à la marge pour complémenter le discours ou les exemples de Valentin. Trois des stagiaires ayant été «condamnés " par un juge au retrait du permis de conduire pour récidives de conduite en état d'ivresse étaient également dans l'obligation d'assister à ce stage. Il est à noter un fait particulier : lors du tour de table qui s'est fait dans un sens de rotation classique, un stagiaire n'a pas été interrogé, il l'a été lors du retour de la pause. Cette anecdote m'avait interrogée, la réponse donnée par Valentin lors de l'autoconfrontation a révélé une stratégie réfléchie liée à l'état de dépendance à l'alcool de ce stagiaire qui était dans l'incapacité de se présenter avant d'avoir « sa dose » prise lors de la pause...

Au début du deuxième jour, nous avons été accueillis par les formateurs qui étaient en train de renseigner les attestations individuelles du stage. Au-delà du fait de préparer effectivement ces attestations, les autoconfrontations ont montré une stratégie destinée à rappeler aux stagiaires éventuellement agressifs que les formateurs avaient un rôle important à jouer sur la récupération de leurs quatre points... L'accidentologie, les risques liés à la vitesse, les temps de réaction ont été animés en majorité par Noémie selon une structure interactive. Les stagiaires en équipes ou individuellement devaient réfléchir, 
échanger, écrire leurs représentations liées aux thèmes abordés : le nombre d'accidents en ville, en rase campagne, par beau temps, les jours de pluie, d'orage, les temps estimés pour arrêter son véhicule à telle ou telle vitesse, dans des circonstances diverses... Après exposés des diverses réponses ou représentations des stagiaires ou des équipes, Noémie présentait des statistiques, illustrées par des exemples régionaux. Valentin intervenait pour complémenter ses propos.

La projection d'un choc d'un véhicule à deux roues contre une automobile, en ville, a suscité des réactions de surprise, liées au fait que chacun réalisait qu'il ne pouvait rien faire en termes de réflexes. La brutalité du choc scénarisée et filmée ne laissait aucun doute sur ce sujet. Il est à noter qu'aucune image sanguinolente " post accident » n'était montrée, le spot s'arrêtait au choc. Les entretiens ont permis aux formateurs de s'exprimer sur des expériences de partenaires qui « apportaient des films réels gores pour les montrer aux stagiaires ", et qui ont généré des conflits entre eux...

28 La remise des attestations en fin de stage n'a suscité aucune réaction particulière, sauf peut-être quelques regards particuliers jetés aux formateurs en sortant, mais peut-être était-ce une interprétation de notre part...

\section{Les cadres conceptuels} transformations respectives de chacun, du dispositif prescrit et les ajustements opérés pendant la situation de formation.

Il y a transformation du dispositif prescrit, en un dispositif qui semble, pour les formateurs plus adaptés au public qui est présent dans la situation. De plus, il apparaît que les formateurs ne se rencontrent pas, ou très peu, pour préparer des sessions particulières. Les transformations du dispositif semblent donc s'opérer non pas en amont, mais pendant les deux jours de formation. Ces adaptations émanent en grande partie du nombre important d'aléas et d'imprévus qui surviennent, de la part des stagiaires, qui pour les formateurs montrent l'inadaptation du dispositif prescrit et qui débouchent quelquefois sur des conflits ouverts ou des exclusions du stage. Ainsi, nous avons fait l'hypothèse que les genèses instrumentales pouvaient permettre d'éclairer ces transformations $\mathrm{du}$ dispositif prescrit pendant la formation, et les concepts pragmatiques, la cohabitation du binôme procédant d'une part à une co-animation, et d'autre part à des transformations individuelles pendant la situation, plus ou moins acceptées par l'autre membre du binôme. L'autre hypothèse que nous avons construite, partait du fait que ces transformations du dispositif prescrit étaient générées par les imprévus surgissant pendant la situation et ainsi exigeaient des ajustements à l'évolution de la situation. Ainsi, nous avons mobilisé quatre cadres conceptuels

31 Le premier cadre mobilisé dans cette recherche est celui de la théorie de l'activité étendue de Rabardel $(1995,2002)$ part du principe que toute activité est médiatisée par des artefacts. Cette théorie est inspirée de la médiation de l'activité humaine par des outils et des symboles de Vygotski (1930/1985). Dans ce cadre théorique, pour qu'un 
artefact devienne un instrument médiateur pour un sujet, il est nécessaire que deux processus soient effectués :

- vers le sujet: l'artefact va modifier les schèmes de l'utilisateur. Cette assimilation de l'artefact et cette accommodation des schèmes est appelée instrumentation

- vers l'artefact : le sujet va prendre en compte les fonctions de l'artefact, mais va également lui en attribuer d'autres qui n'étaient pas prévues lors de sa conception.

C'est ce double processus que Rabardel appelle genèse instrumentale. Cette approche de l'activité médiatisée, suppose donc que l'instrument médiateur est une unité mixte composée d'un artefact matériel ou symbolique et de schèmes d'utilisation qui lui sont associés par l'utilisateur. Trois orientations de l'activité médiatisée par les artefacts sont envisagées : la médiation vers l'objet de l'activité, la médiation vers le sujet lui-même, la médiation vers les autres sujets. Dans le cas du stage de récupération de points, les trois orientations peuvent être envisagées. Dans notre analyse, nous avons mobilisé ce cadre en considérant que le dispositif "stage" est un artefact, qui, du point de vue des formateurs, devient un instrument. Il s'agit alors de cerner ce qui relève d'un processus d'instrumentation, consistant à modifier les schèmes des formateurs, et ce qui relève d'un processus d'instrumentalisation consistant pour le formateur, à donner au dispositif de nouvelles fonctions. Nous aurions pu envisager le point de vue des stagiaires et nous intéresser à leurs propres genèses instrumentales, mais cela n'a pas été pris en compte dans cette recherche.

Nous avons également mobilisé le modèle des gestes professionnels d'ajustement (Bucheton, 2009) qui a été élaboré dans le but d'analyser les macro-préoccupations d'enseignants et de formateurs dans une situation éducative et/ou de formation. Afin de s'ajuster aux imprévus ou aux fluctuations de la situation, le formateur génère quatre types de macro-préoccupations, qu'il va essayer de " traduire » en actions concrètes dans la situation, pour obtenir les effets qui pourraient satisfaire ses visées. Ainsi, ces préoccupations vont se décliner en combinaisons de gestes professionnels d'ajustement (Jean, 2009) situées et singulières en fonction des particularités de la situation et des décisions qu'il va prendre pour mettre en œuvre et optimiser ses macro-préoccupations. Autour de l'objet de savoir à faire acquérir aux stagiaires, le formateur va se préoccuper de créer de l'atmosphère, du tissage, de l'étayage et du pilotage (Bucheton, 2009). L'atmosphère correspond à l'ambiance que l'on veut donner et maintenir dans la situation de formation, aux interactions autorisées ou pas, à la place laissée à la parole des stagiaires bref... à l'atmosphère qui va être construite par le formateur, et dans notre cas, à l'atmosphère qui va être co-construite par le binôme formateur. À noter cependant un geste de contre-atmosphère correspond à couper la parole, à ne pas laisser de place à la parole des stagiaires. Le tissage correspond aux liens que le formateur a l'intention d'élaborer avec tout ce qui n'est pas « ici et maintenant » dans la situation de formation. Cela correspond par exemple pour notre cas, à «Vous voyez la signalisation routière au carrefour du Rieucoulon? Et bien vous ne verrez pas de Stop alors que ce croisement est dangereux » ou encore "Demain, nous, aborderons les distances d'arrêt ». Le pilotage correspond à la gestion spatio-temporelle de la situation. Ainsi, la gestion du temps, du matériel utilisé, de la disposition des stagiaires dans la salle, les placements et déplacements des formateurs, reflètent cette macro-préoccupation. Enfin l'étayage, notion empruntée à Brunner (1983), représente les gestes qui ont pour visée de faire faire, faire dire, faire apprendre aux stagiaires et la façon de le faire. Ces macro-préoccupations, sont des visées globales des formateurs, qui vont s'actualiser dans la situation par des combinaisons de 
gestes professionnels d'ajustement. Nous allons repérer par exemple des combinaisons " tissage-étayage-atmosphère », ou atmosphère-étayage-pilotage », essayant de traduire les macro-préoccupations des formateurs par des gestes concrets en situation. Certaines vont lui permettre d'atteindre les visées recherchées, d'autres non. Ce ne sont pas les résultats visibles dans la situation qui nous intéressent ici, en tant qu'actualisation des macro-préoccupations, mais les macro-préoccupations elles-mêmes, ce qui nécessite les points de vue des acteurs

Pour les raisons que nous expliquons plus haut, nous avons prêté une attention particulière aux imprévus qui surgissent dans une situation de formation, et vont pousser les formateurs à s'ajuster à la situation. Le concept d'imprévu dans une situation d'éducation ou de formation a été développé entre autres par Perrenoud (1999) et Hubert et Chautard (2001). Jean (2012), dans un contexte de formation, souligne que les imprévus peuvent provenir de trois origines : les stagiaires (ce sont les plus importants ici), les formateurs eux-mêmes (le fait d'être en binôme les multiplie), et le monde extérieur à la situation (une personne qui rentre, un bruit de freinage de véhicule, des perturbations sonores extérieures...). Le traitement d'un imprévu, à partir du moment où il a été perçu, peut s'analyser selon un choix entre deux alternées. La première alternative consiste à feindre de ne pas avoir perçu et d'essayer de continuer la formation sans réaction particulière. C'est en quelque sorte un déni de l'imprévu, ou en d'autres termes ignorer sa survenue. La deuxième alternative consiste à transformer l'imprévu en "périphénomène ", c'est-à-dire à lui donner volontairement de l'importance à la vue de tout ou partie de l'assemblée des stagiaires. Ce traitement, la plupart du temps, a pour visée de se servir de l'imprévu à des fins pédagogiques ou didactiques. Le choix entre les deux alternatives relève d'une mise en balance entre ce que peut risquer ou ce que peut gagner la situation de formation entre ignorer l'imprévu ou s'en servir. Un troisième élément du triptyque est l'événement, pris au sens de Ricœur (1991), c'est-à-dire quelque chose dont on ne pouvait pas prévoir la survenue, qui sort du champ des possibles d'un sujet, ou dont le sujet est incapable d'en comprendre la signification. Ainsi, il faut reconnaître la relativité de l'imprévu, selon les personnes qui le perçoivent. Pour certains, ce qui se produit là n'est pas un véritable imprévu (Jean, 2012) parle ici " d'imprévus-prévus ») alors que pour d'autres c'en est un et pour d'autres encore ce sera un événement. Le point de vue de l'acteur (de chaque acteur du binôme ici) est donc indispensable, pour qualifier d'imprévus, ce qui s'est passé pendant le stage. Ceci peut nous amener à une réaction de stagiaire qualifiée d'imprévu par un formateur du binôme, et d'événement par l'autre, avec toutes les combinaisons possibles des éléments du triptyque présenté ci-dessus. Comme il a été précisé plus haut, les stages de récupération de points sont potentiellement générateurs d'imprévus liés à des stagiaires chez qui une agressivité importante est souvent présente. Mais le fait d'assurer la formation en binôme sans nécessairement d'accord entre les deux formateurs sur le déroulement, les contenus et leur rapport aux prescriptions, apporte également une potentialité importante d'imprévus liés aux formateurs. Un nombre non négligeable de ces imprévus sont déjà en eux-mêmes ostentatoires, ce qui limite la part du déni d'imprévus du triptyque présenté : il est difficile de feindre de ne pas percevoir une agression contre soi, même si certains formateurs y parviennent. 


\section{Les concepts pragmatiques (Pastré, 2011)}

Comme nous l'avons précisé plus haut, les transformations qu'opèrent pendant la situation, chacun des formateurs du binôme, sont pour la plupart acceptées par l'autre. Cependant certaines génèrent des tensions perceptibles ou pas. Ces transformations partent d'un diagnostic d'une situation qui ne serait pas adaptée aux stagiaires ou aux visées du formateur. Les concepts pragmatiques (Pastré, 2011) nous sont apparus comme particulièrement adéquats pour rendre compte de ce qui se joue dans la situation. De plus, chacun des formateurs a une visée à court terme : assurer la formation dans des conditions acceptables, et une visée à long terme : pendant la formation, faire en sorte de changer le comportement des conducteurs infractionnistes qui sont présents. Il y aurait donc des transformations liées aux deux types de visées, selon les représentations que se fait le formateur de la situation qu'il est en train de vivre. Nous pouvons supposer que ces transformations émanent en partie, de choix de règles d'actions estimées pertinentes en regard à la situation. Il apparaît que seules, ces règles d'action sont insuffisantes pour permettre d'orienter une action, dans un environnement complexe, pour permettre de diagnostiquer la situation et donc de choisir entre plusieurs règles d'action. Pastré apporte la notion de concept pragmatique (2011), permettant de conceptualiser la situation, de s'en faire une représentation au sens d'image opérative d'Ochanine (1978) ou au sens de représentation fonctionnelle de Leplat (1985). Il s'agit en fait de comprendre pour agir, c'est-à-dire de choisir une règle d'action qui paraît pertinente pour la situation, en fonction de la représentation élaborée par l'opérateur à l'aide des concepts pragmatiques.

Les savoirs de référence sont définis comme un ensemble de savoirs reconnus par la profession sur les objets du domaine, et de « savoirs en actes efficaces » manifestés dans les pratiques professionnelles (Rogalski \& Samurçay, 1994 ; Samurçay \& Rogalski, 1992). Cela revient à identifier les invariants conceptuels et stratégiques qui organisant l'activité efficace pour le traitement d'une classe de situations. Les concepts pragmatiques sont définis comme ces unités opérationnelles organisatrices et constitutives des savoirs de référence.

Les concepts pragmatiques ont un double rôle: "d'une part ils organisent l'action efficace en permettant un couplage fort entre les prises d'informations effectuées par le sujet et les opérations qu'il exécute; d'autre part ils permettent à sa représentation (fonctionnelle) de se structurer, en mettant l'accent sur les relations essentielles existant entre les variables de la situation » (Pastré 1999, p. 16). Du coup, on peut les considérer soit comme des invariants de l'action et cela renvoie au concept de schème comme " organisation invariante de la conduite "; soit comme des invariants conceptuels qui guident l'activité au niveau de la représentation, autrement dit comme des descripteurs qui décrivent la situation de référence selon une de ses dimensions essentielles.

Les concepts mobilisés dans l'action ont une double dimension: une dimension épistémique, comme éléments de connaissance du fonctionnement d'une situation ; une dimension pragmatique, comme moyen de prélèvement de l'information pertinente pour l'action.

Un concept pragmatique est donc une dimension de la situation, dont l'évaluation permet de porter un diagnostic de fonctionnement de l'ensemble de la situation. Il est constitué de deux éléments : un indice observable dans la situation, qui permet une évaluation du 
phénomène perçu; et un signifié, qui est la dimension abstraite qui a été retenue (attitude agressive, motivation, inattention...). En tant qu'indicateur, il permet de porter un diagnostic de la situation. En tant que variable, il constitue un des éléments de connaissance de la situation.

Nous pouvons donc déceler deux types de concepts pragmatiques, le premier type concerne le diagnostic d'une situation de formation qui se déroule dans le calme et la sérénité en maintenant "la paix sociale », le second type concerne le diagnostic d'une situation qui va permettre de faire prendre conscience aux stagiaires qu'ils doivent changer de comportement en tant que conducteurs.

\section{Des genèses instrumentales}

\subsection{Les redéfinitions des tâches}

Les prescriptions nationales attribuent à ces stages des visées de responsabilisation des conducteurs sur la route. Le «bref historique des stages de sensibilisation à la sécurité routière » présenté ci-dessus en montre les objectifs principaux. Les formateurs tiennent un discours plus précis sur les objectifs du stage. Il s'agit de faire prendre conscience aux conducteurs infractionnistes, des dangers qu'ils ne soupçonnent pas. Par exemple des temps de perceptions et de réponses des êtres humains devant un danger: les conducteurs qui se croient plus performants que les autres se trompent et l'invulnérabilité que certains s'attribuent est utopique. Le rapport à l'alcool est également visé pour les comportements qui lui sont associés sur la route, tant au niveau social qu'au niveau des performances de conduite. Le binôme redéfinit également les tâches selon les convictions de chaque animateur. Lors des entretiens d'autoconfrontation, ils disent ne pas se rencontrer pour la préparation des stages. Ils connaissent globalement les valeurs de l'autre, et procèdent à des arrangements durant les deux journées, soit pendant les activités des stagiaires, soit pendant les pauses. Ils redéfinissent la tâche de deux manières, d'une part, en modifiant les méthodes pédagogiques prescrites, d'autre part en remplaçant les contenus prévus par des messages qu'ils veulent faire passer, messages, dont nous faisons l'hypothèse qu'ils sont étroitement liés à leurs valeurs, logiques profondes, conception et vision du monde qui les entoure. Il était donc important pour nous, d'une part de renseigner les redéfinitions des tâches, permettant ainsi de comprendre les intentions, les préoccupations, les visées redéfinies par chacun des formateurs selon ses convictions, et d'autre part, de les mettre en relation avec ce qui s'est passé réellement, en tenant compte des réactions des divers acteurs de la situation et des ajustements et compromis en acte que chacun a fait, des genèses instrumentales opérées. Les redéfinitions des tâches sont exprimées par les formateurs en début des autoconfrontations. Nous pouvons remarquer que les messages que chacun veut faire passer pendant le stage sont différents de nature, voire opposés.

Valentin : les messages qu'il veut faire passer au travers du $1^{\circ}$ jour de stage (le tour de table) :

- le rapport à la loi est ce qui fait l'homme pensant,

- la quête du bonheur est vaine dans ce monde,

- la valorisation de l'homme au travers d'une voiture, de sa puissance ou de sa vitesse est vaine : « Nous finissons tous de la même façon : en terre ». 

table):

- elle n'est pas d'accord avec ce rapport aveugle à la loi que veut faire passer Valentin. Il faut questionner la loi et avoir l'esprit critique,

- le rapport à la loi aveugle revient à une soumission. Ce n'est pas acceptable,

- la position de Valentin est une position de lacanien : c'est son profil. «Le tour de table de cette façon, c'est un besoin autant que de sensibiliser les autres à la sécurité routière ».

Nous pouvons relever que les tâches ne sont pas les mêmes pour Noémie et pour Valentin.

\subsection{Les genèses instrumentales repérées}

Nous les avons repérées dans leurs discours sur les traitements des imprévus qu'ils avaient validés. Ces traitements, comme nous l'avons montré (Jean, 2012), sont des révélateurs des valeurs, les logiques profondes, des représentations, mais révèlent également des ajustements dans l'urgence à la situation, au binôme et aux stagiaires. Ils nous renseignent sur les compromis, les stratégies dans l'action, les concessions nécessaires à la tenue du stage, aux relations avec le binôme, les stagiaires et aux changements de comportements visés des conducteurs, qui persistent en toile de fond. Il est important ici, de préciser qu'en prenant en compte la temporalité nécessaire à des genèses instrumentales, Noémie et Valentin travaillent en binôme, depuis quelques années. Toutes leurs interventions ne se font pas dans le même binôme, mais au fil des configurations de stages, ils se retrouvent régulièrement. Dans leur discours sur leur activité, même s'ils reconnaissent avoir une idée des réactions de l'autre, ils affirment que chaque stage est différent du précédent et qu'ils n'ont pas fini de se découvrir. On peut donc raisonnablement supposer que les genèses instrumentales repérées ci-dessous se sont faites sur des temporalités longues et que les processus sont toujours en cours.

Valentin : Nous repérons au moins une genèse instrumentale

Il conçoit et accepte d'animer le stage, bien que pas d'accord avec toutes les prescriptions à condition :

- d'utiliser une journée, pour faire passer le message qu'il estime le plus important («le rapport à la loi, est-ce qui fait l'homme pensant ») au détriment des contenus prescrits et à sa manière (le tour de table), au détriment des cadres théoriques d'apprentissages prescrits (conflits sociocognitifs, socioconstructivisme...). Il attribue des fonctions à l'artefact et opère sur ce dernier des transformations locales ou durables : c'est une instrumentalisation,

- d'appliquer les prescriptions durant la seconde journée et ainsi de déroger à ses convictions pour aborder ce qu'il estime peu important et peu efficace. Pendant cette journée, suivre le dispositif prescrit l'oblige à modifier ses schèmes d'action : c'est une instrumentation.

Il s'agit bien ici d'une genèse instrumentale par la création d'un instrument à partir de l'artefact constitué par le dispositif et ses prescriptions.

Noémie : Nous repérons au moins une genèse instrumentale

50

Elle conçoit et accepte d'animer le stage, bien que pas d'accord avec toutes les prescriptions. Cependant, elle s'arrange pour reconfigurer, à la marge, la façon dont Valentin a configuré la prescription. 
51 La position des «co-animateurs psy n'est pas confortable». Au vu du turn-over chez ces spécialistes théoriciens, et de ce que « peut apporter un type comme Valentin »,

- Elle laisse faire Valentin dans «son discours de prêcheur », parce que " c'est son truc et que finalement ça marche quand même », parce qu'elle le connaît, " même si c'est en dehors des clous, il passe bien et son discours porte ». Elle n'est pas d'accord avec l'axe théorique sur lequel est fondé le stage de $2^{\text {ème }}$ génération. Pour elle, ce modèle a été conçu et validé avec un public de personnes volontaires, ayant accepté de se déplacer en termes de posture de conducteur, alors que les stages de récupération de points ne réunissent pas le même public. La motivation y est totalement utopique, car les participants ont perdu tous leurs points et redoutent l'annulation de leur permis. Nous pouvons parler d'instrumentation dans le sens où l'artefact ainsi modifié agit sur ses schèmes d'action.

- Mais dès qu'elle le peut, Noémie intervient pour moduler le propos de Valentin et montrer qu'il faut être critique et qu'on peut réfléchir en conduisant. De plus, au vu de ce qu'apporte un « type comme Valentin, ça vaut le coup de le laisser s'éloigner des clous ». Mais en milieu d'après-midi, si seuls 3 ou 4 stagiaires sur seize se sont présentés dans le tour de table, elle lui demande de « booster un peu ». Une journée d'accord à condition que le tour de table soit terminé à 16 heures. Ces fonctions nouvelles attribuées à l'artefact sont une instrumentalisation.

Il s'agit bien ici d'une genèse instrumentale par la création d'un instrument à partir de l'artefact constitué par le dispositif et ses prescriptions

Ces deux genèses instrumentales aboutissent, in fine à un seul et même dispositif : le stage vécu et assuré devant les stagiaires. Il s'agit bien d'après Flocher (2005), du fruit de l'activité située et finalisée des formateurs qui se concrétise dans l'instrument qu'ils ont développé au cours du processus d'appropriation. Le fait qu'ils n'aient pas échangé avant le stage, pour en construire les contenus, et donc le faire ici et maintenant, dans l'action, nous fait supposer qu'il s'agirait d'une conception commune dans l'usage d'un instrument à partir d'un artefact (la prescription) qualifié par les deux formateurs de «en dehors des clous» en tant que dispositif de formation pour récupérer des points du permis de conduire.

\subsection{Les concepts pragmatiques repérés}

Pour chacun des co-animateurs, nous avons repéré deux types de concepts pragmatiques, liés à des actions au sein d'une activité visant soit du court terme soit du long terme. C'est-à-dire visant d'une part des situations d'apprentissages de stagiaires en tant que personnes dans le stage, et d'autre part, visant des situations de transformations de comportements des stagiaires en tant que conducteurs lorsqu'ils auront terminé le stage : le type 1 consiste à animer un stage, à générer des apprentissages à court terme : les deux jours de stage ; le type 2 consiste à modifier des postures de conduite automobile des stagiaires, à long terme (« l'après-stage »).

Valentin : Type 1 : animer le stage, générer des apprentissages et des déplacements

- Les caractères particuliers des stagiaires se détectent au travers de leurs réponses aux deux questions qui leur sont posées et de leurs réactions aux propos qu'il tient suite à leurs réponses. Les détections de ces caractères particuliers permettent de se faire une idée précise du public du stage.

- Les réactions d'acquiescement, de réprobation ou d'étonnement des stagiaires face à des études de signalisations routières locales, réelles, que les stagiaires de la région connaissent, 
et pratiquent quotidiennement, montrent qu'il y a production d'apprentissages et de déplacements.

- Les postures agressives diminuent lorsqu'on évoque l'attestation d'assiduité au stage qui sera remise à la fin du stage. Il faut donc le rappeler de temps en temps.

- La diminution de réactions des stagiaires face à la répétition régulière d'un message durant une journée permet déceler l'adhésion au contenu de ce message.

\section{Noémie : Type 2 : La posture du conducteur sur la route}

- Une bonne situation de conduite se repère au fait que le conducteur se pose des questions sur la réglementation adoptée, qu'il réfléchit aux raisons de la présence de signalisation routière. "Un conducteur ne doit pas être soumis ";

- une situation correcte de conduite à vitesse limitée, se repère lorsque le conducteur roule à la limite de la verbalisation. : "On peut afficher $93 \mathrm{~km} / \mathrm{h}$ sur le régulateur lorsque la vitesse est limitée à $90 \mathrm{~km} / \mathrm{h}$ ». Sur ce point, on repère deux points de vue différents des formateurs : Valentin se réfère à la sécurité routière, alors que Noémie parle d'infraction qui conduit à des procès verbaux et des retraits de points. Ce sont deux visions de la formation différentes et surtout des objectifs liés à ce stage.

\section{Quelles combinaisons de gestes professionnels d'ajustement aux imprévus?}

Tout d'abord, le nombre d'imprévus survenus pendant les deux jours de stage ne sont pas aussi nombreux que l'on pouvait s'imaginer. Nous avons comptabilisé 0,2 imprévu/ minute (à titre comparatif, en situation de classe en collège par exemple, la moyenne se situe à 1,5 imprévus/minute). Durant les 2 journées de stage, sans compter les discussions pendant les pauses, 96 imprévus ont donc été repérés par l'analyste et confirmés par les 
formateurs. Il s'agit d'un maximum, car pour Valentin, il n'en reconnait que 70, alors que Noémie en reconnaît 96 . Sur ces 96 imprévus, Valentin considère que 36 sont des imprévus-prévus, donc sans trop de surprise pour lui, mais nécessitant un ajustement. Noémie repère 47 imprévus-prévus, donc déjà vus lors d'autres stages.

Les gestes professionnels d'ajustement sont très liés à chacun des formateurs. On peut faire l'hypothèse que le statut de l'animateur, en tant que psychologue ou de formateur BAFM, joue un rôle important.

61 Sur les deux journées, les gestes et combinaisons de gestes d'ajustement montrent des différences significatives entre chacun des formateurs.

Figure 1 : Valentin - Combinaison prégnante : Étayage - contre-atmosphère - tissage.

Figure 1: Valentin - The most important combination of professional gestures - Scaffolding - gesture of denial - Weaving

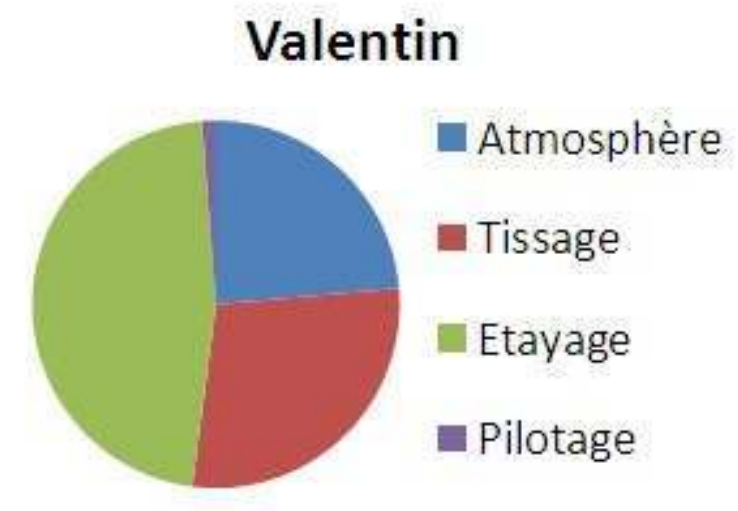

Figure 2 : Noémie - Compbinaison prégante : Atmosphère-pilotage-étayage. Figure 2: Noémie- The most important combination of professional gestures - Atmosphere-pilotingscaffolding

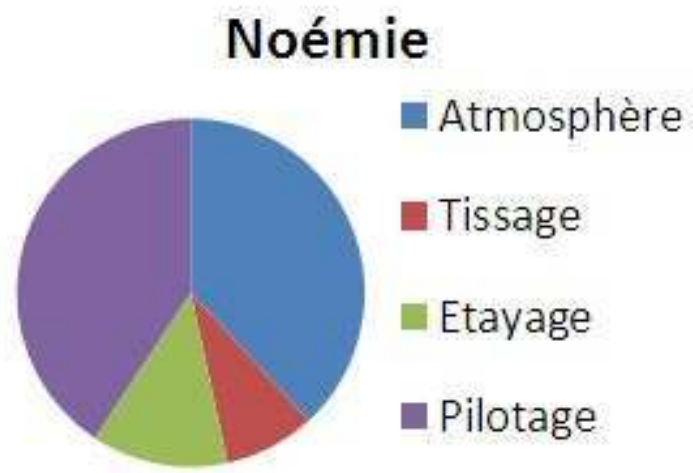

62 La prédominance de contre-atmosphère pour Valentin correspond à la journée tour de table qu'il consacre à son discours de répétition de messages, à partir des réponses que les stagiaires donnent aux trois questions qu'il a posées de manière générique. Ses ajustements aux imprévus provenant de contestations de stagiaires consistent souvent à reprendre le plus rapidement possible la parole afin d'argumenter dans son sens. 


\section{Discussion} c'est la répétition des messages qui, d'après lui, est efficace. Son temps de parole est très important au point de ressembler à un monologue. La grande majorité des gestes professionnels d'ajustement de contre-atmosphère, montre le peu de place laissé à la parole des stagiaires. Après le geste d'étayage, dû aux nombreuses démonstrations des messages qu'il veut transmettre, le geste de tissage semble être un geste professionnel d'ajustement prédominant chez Valentin. Sa conception de l'apprentissage place le formateur au centre des discours dans une approche frontale, mais qui semble fonctionner en termes de tenue de stage devant des stagiaires contraints d'y participer. La préoccupation de Valentin consistant à essayer d'expliquer les raisons à la présence de signalisations routières réelles, connues géographiquement par les stagiaires, induit un taux d'écoute élevé. Cependant, les réactions des stagiaires devant certaines de ses explications montrent également des diversités de points de vue et des départs de débats sur la loi et son application. Dans les entretiens d'autoconfrontation, Valentin précise que certains de ses propos sont des provocations volontaires. Ceci montre que si l'argumentation de Valentin est plongeante, si le déni de certaines interventions des stagiaires est présent, ses explicitations tentent de provoquer des conflits cognitifs ou sociocognitifs chez les stagiaires. Son approche est donc relativement mêlée de béhaviorisme et de socioconstructivisme. Il est également intéressant de noter que les contenus qu'il aborde relèvent de visions du monde particulières (« on finit tous de la même façon, sous terre ", «nul besoin de recherche de la toute puissance à travers les voitures »...) et de concepts et auteurs philosophiques (il parle explicitement de la jouissance, de l'homme pensant, de Platon, d'Aristote...). Enfin, les contenus sont relativement assimilés, car, pendant le deuxième jour, certains stagiaires ont mobilisé des ressources acquises lors du premier jour.

Noémie facilite la mise en activité des stagiaires en les plaçant dans des activités de débats, de confrontations d'opinions, de réflexions sur des questionnements relatifs à la conduite routière. Les aspects théoriques sont apportés après l'expression des stagiaires devant des situations pour lesquelles ils sont mis en situation de résoudre les difficultés. Les temps de paroles sont plutôt équilibrés entre stagiaires et formatrice, ce qui montre la place accordée au point de vue des autres. En termes d'organisation pédagogique, la constitution de groupes réfléchissant et échangeant sur les situations proposées, le temps consacré à ces activités, montre une conception de l'apprentissage relevant du socioconstructivisme. On comprend aisément que l'ambiance du premier jour n'a pas été de même nature que celle du deuxième jour. Les échanges entre les stagiaires, beaucoup plus nombreux le deuxième jour pendant les pauses, ont également tendance à le montrer, même si la prolongation de la promiscuité peut également augmenter la communication entre stagiaires. Mêlées à quelques conversations de politesse, bon nombre de ces dernières portaient sur le domaine de la conduite automobile.

67 Ces différences entre les deux formateurs du binôme, ont en définitive provoqué de la complémentarité, tant dans les approches pédagogiques, que dans les mises en activité 
des stagiaires. Cette complémentarité n'est pas exprimée clairement dans les propos des formateurs lors des autoconfrontations. Leurs différences sont certes connues, car, rappelons-le, les deux formateurs se connaissent depuis longtemps. Cependant, c'est plus en termes de concessions faites de l'un vers l'autre qu'elles sont exprimées. Le stage devient un lieu de concessions, dans lequel ils se laissent l'un après l'autre des temps pour le faire évoluer chacun à leur manière. On note très clairement un effacement de Noémie le premier jour au profit de Valentin, et l'inverse le deuxième jour. Le stage, en théorie fondé sur des prescriptions serrées, comme nous l'avons vu, se construit d'une tout autre manière, pour servir, alternativement, les conceptions de l'apprentissage de chacun des formateurs du binôme. Cependant, nous faisons l'hypothèse que cette co-construction du stage au travers des genèses instrumentales présentées plus haut, relève de conceptions dans l'usage (Rabardel, 1995), que nous pourrions qualifier d'alternées : chaque formateur réalise la tâche qu'il estime être à faire, laisse la main à l'autre, qui procède de même. Dans ce sens, nous considérons avec Folcher (2005, p. 190), que « la conception dans l'usage est le fruit de l'activité d'utilisateurs située et finalisée qui se concrétise dans les instruments qu'ils développent au cours des processus d'appropriation. L'appropriation et la constitution d'instruments dans l'usage sont mobilisées au cours d'une genèse instrumentale qui transforme à la fois l'organisation de l'activité des sujets, les tâches qu'ils s'attribuent, les activités qu'ils réalisent et les caractéristiques de l'artefact ». Ces concrétisations des genèses instrumentales, relèvent selon nous, de conceptions alternées dans l'usage. La définition que propose Folcher de la conception dans l'usage nous conforte dans cette hypothèse. L'instrument qui se développe, alternativement pour chacun des formateurs, à partir du dispositif de formation de stage de récupération de points, considéré au départ, comme un artefact a tendance à le montrer. Nous pourrions presque considérer que deux instruments différents sont développés en parallèle par chaque formateur, à partir d'un même artefact. L'ensemble du dispositif sur des deux jours, est donc co-construit par le binôme de formateurs, en s'écartant des prescriptions tant nationales, que locales (celles de l'association)

Nous pourrions étendre cette hypothèse : l'hétérogénéité du public de chaque session de formation constitue une caractéristique fondamentale de ce type de sensibilisation à la conduite routière. Dans ce sens, lors de la mise en œuvre de ce dispositif de formation par les binômes de formateurs devant les stagiaires, une co-animation se met en place et en œuvre et elle inclurait :

- d'une part, des diagnostics des situations de formation vécues par chacun des formateurs.

- d'autre part, des valeurs, des logiques profondes, des concepts pragmatiques et des genèses instrumentales relatifs à chacun des formateurs du binôme. Ces éléments dialoguant, rentrant en conflit, coexistant, et construisant à chaque session un nouvel instrument qui peut être éphémère le temps de la session et ne plus se renouveler, même si le binôme se retrouve constitué, comme se reproduire, et véhiculer les messages que chacun estime essentiels.

69 Ainsi, en termes de conceptions de dispositifs de formation mis en œuvre en co-animation par deux types de formateurs, il nous semble important de considérer ce qui ce passe dans le type de situation que nous venons de décrire. Ces conceptions alternées dans l'usage, ces développements d'instruments différents à partir d'un même dispositif, pourraient être admis non seulement dès la phase de conception de dispositifs, dans le but de laisser des marges de manœuvre aux prescriptions de formation, mais également dans les consignes données aux «inspecteurs", qui aux dires des formateurs, sont 
proches de contrôles de conformités par rapport aux prescriptions. Mais l'un des éléments qui nous semble le plus important à prendre en compte réside, à notre avis, dans la formation de formateurs qu'il faudrait mettre en œuvre. Les formateurs que nous avons interrogés ont largement dépassé le temps qu'ils avaient décidé de consacrer à notre recherche. Les entretiens d'autoconfrontation leur permettant de verbaliser leur activité les a d'abord surpris, puis intéressés, au point de proposer de les mener avec d'autres formateurs sur d'autres sessions.

\section{Conclusion}

En conclusion, cette recherche dont il faut relativiser une partie des résultats présentés, car situés sur un seul stage, nous a fait découvrir un monde de la formation singulier. Les formateurs qui y interviennent, du moins ceux que nous avons rencontrés, sont investis d'une mission qu'ils portent à un haut niveau, car elle permet de diminuer le nombre de vies fauchées sur la route dont on connaît malheureusement les chiffres toujours alarmants même si la tendance est à une nette baisse depuis une dizaine d'années. Les publics à qui ils ont à faire sont pour la plupart persuadés que ce sont les autres qui commettent des imprudences et ils ne comprennent pas leur présence dans ces stages. Ces formateurs œuvrent donc dans un univers de résistances, de conflits, de violence parfois, dont les enjeux dépassent le stage et les formateurs eux-mêmes.

71 Nous avons montré que ce qui se joue essentiellement dans le stage que nous avons étudié, relève de conceptions alternées dans l'usage de la part des deux formateurs en coanimation. Les genèses instrumentales opérées sur le dispositif permettent à chacun des formateurs du binôme de faire passer aux stagiaires leurs valeurs, leurs logiques profondes, leur vision du monde, leurs manières de se comporter sur la route et vis-à-vis de la loi. Ces éléments sont très liés à la personne et aboutissent donc à des tensions, des échanges, des porosités pendant ces stages soit avec ceux des autres formateurs soit avec ceux des stagiaires. Les problèmes entre formateurs sont en grande partie liés à ces divergences de points de vue. La diversité des formateurs recrutés, tant chez les psychologues que chez les formateurs BAFM, génère autant de diversité de points de vue sur la conduite de stage mais également sur les comportements d'usagers de la route. Le turn-over constaté, accentue certainement cette diversité. Ce n'est pas tant la diversité en tant que telle, qui pose problème, mais les incompatibilités des points de vue qui se révèlent pendant le stage et qui reposent, en partie, sur des valeurs. Ces incompatibilités pourraient être atténuées en amont. Dans cette perspective, la formation de formateurs nous semble indispensable pour actualiser, échanger, construire et argumenter ces différents points de vue. Il est bien entendu que cela va au-delà d'informations sur les prescriptions, les législations relatives à la conduite automobile, les approches théoriques, qui restent indispensables. Les analyses effectuées lors de cette recherche pourraient servir d'appui sur des formations qui prendraient pour thème, des analyses de situations réelles, avec les points de vue des acteurs. Ainsi, les divergences de points de vue entre formateurs soit sur des traitements d'imprévus survenant pendant la situation, soit sur des conceptions alternées dans l'usage, pourraient être élaborées. Ainsi, en prenant les précautions habituelles d'anonymat dans les situations analysées, une formation par la recherche serait peut-être une piste intéressante pour cet univers de la formation. Dans cette idée, il aurait été intéressant de mener une étude analysant les points de vue des stagiaires sur le stage, et les modifications de comportements et de 
visions de la route qu'il pourrait avoir engendrées. Ce n'était pas le but de cette recherche, mais cela peut être une piste intéressante pour les nouvelles générations de stage qui vont se mettre en place et ainsi, permettre aux formateurs d'avoir un retour, si minime soit-il sur les effets des stages qu'ils animent.

Nous pourrions également faire des liens avec d'autres domaines de formation ou d'éducation. Les situations de co-animations dans des formations professionnelles ou scolaires, pendant lesquelles les publics ne sont pas particulièrement présents de manière spontanée, sont nombreuses. Nous pensons par exemple aux lycées professionnels, aux formations dans l'agriculture ou aux divers dispositifs en collèges et en lycées, mais il y en a bien d'autres. Dans ces domaines, des professionnels d'origines diverses, co-animent des situations d'apprentissage, en théorie, pour apporter une certaine complémentarité correspondant à leurs spécialisations. En réalité ces co-animations ne se font pas toutes sereinement et les visées ne sont pas toujours atteintes. Ce type de situations de formation que nous présentons, dans laquelle tous les stagiaires sont contraints d'assister sous peine de conséquences quelquefois graves, constitue à notre avis, une classe de situations de formation, emblématique pour la co-animation, et extrême en ce qui concerne le type de public. Nous pensons donc que cette étude pourrait apporter des éléments transposables dans des situations moins contraintes, ou permettre de donner des pistes pour éclairer les dysfonctionnements.

Enfin, le hasard a fait que depuis notre recherche, la réalisatrice Coline Serrault a présenté sur les écrans son film (2014) «Tout est permis » constitué d'extraits de vidéos filmées pendant ces stages. L'ambiance, les discours, les valeurs, les personnalités qui y sont montrés reflètent parfaitement ce que nous avons constaté et ressenti lors de cette recherche.

En tout état de cause, force est de constater que depuis notre participation à ce stage, notre façon de conduire a changé...

\section{BIBLIOGRAPHIE}

Béguin, P., \& Cerf, M. (2004). Formes et enjeux de l'analyse de l'activité pour la conception des systèmes de travail. @ctivités, 1(1), 54-71. <http://activites.revues.org/1156>

Bucheton, D. (2009, Ed..). L'agir enseignant : des gestes professionnels ajustés. Toulouse : Octarès.

Derouet, J.-L. (1988). Désaccords et arrangements dans les collèges (1981-1985). Eléments pour une sociologie des établissements scolaires. Revue Française de Pédagogie, nº 83, 5-22.

Folcher, V. (2005). De la conception pour l'usage au développement de ressources pour l'activité. In P. Rabardel \& P. Pastré (Eds.), Modèles du sujet pour la conception. Dialectiques activités développement. (pp. 189-210). Toulouse : Octarès.

Huber, M., \& Chautard, P. (2001). Le savoir caché des enseignants. Paris : l'Harmattan. 
Jean, A. (2009). Le cas des gestes professionnels convoqués par les jeunes enseignants de technologie pour traiter les imprévus surgissant en classe. Les Cahiers des Études et de la Recherche en Formation, Éducation et Enseignement, nํำ 26, 165-183.

Jean, A. (2012). L'analyse des imprévus et des événements dans le cadre de la cohérence entre théorie et pratique. In Paquay, L., Altet, M., \& Perrenoud, P. La formation des enseignants : en quête de cohérence (pp. 69-84). Bruxelles: De Boeck Université

Keskinen, E. (1996), Why Do Young Drivers Have More Accidents? Bundesanstalt für Strassenwesen (Bast). Berisch Bladbach: Germany.

Leplat, J. (1985). Les représentations fonctionnelles dans le travail. Psychologie Française, 30(3-4), 269-276.

Ochanine, D. (1978). Le role des images opératives dans la régulation des activités de travail. Psychologie et Éducation, 2, 63-72.

Pastré, P. (1999). La conceptualisation dans l'action : bilan et nouvelles perspectives. Éducation permanente, 139, 13-37.

Pastré, P. (2011). La didactique professionnelle. Approche anthropologique du développement des adultes. Paris : PUF.

Perrenoud, P. (1999). Gestion de l'imprévu, analyse de l'action et construction de compétences. Éducation permanente, 140, 123-144.

Prochaska, J.O., Di-Clemente, C.C., \& Norcross, J.C. (1992). In search of how people change: Applications to addictive behaviors. American Psychologist, 47(9), 1102-1114.

Rabardel, R. (1995). Les hommes et les technologies. Approche Cognitive des instruments contemporains. Paris : Armand Colin.

Rabardel, P. (2002). Le langage comme instrument ? Eléments pour une théorie instrumentale élargie. In Y. Clot (Ed.). Avec Vygostki. (pp. 241-265). Paris : La dispute.

Ricœur, P., (1991). Evénement et sens. Raisons pratiques, L'événement en perspective, 2, 41-56.

Rogalski, J., \& Samurçay, R. (4994). Modélisation d'un savoir de référence et transposition didactique dans la formation de professionnels de haut niveau. In G. Arsac (Ed.), La transposition didactique à l'épreuve (pp. 35-71). Paris : La pensée sauvage.

Samurçay, R., \& Rogalski, J. (1992). Formation aux activités de gestion d'environnements dynamiques : concepts et méthodes. Éducation permanente, 111, 227-242.

Vygotski, L.S. (1930/1985). La méthode instrumentale en psychologie. In B. Schneuwly \& J.P. Bronckart (Eds.), Vygotsky aujourdhui (pp. 19-48). Neuchâtel : Delachaux et Niestlé.

Textes de lois et arrêtés :

Loi no 89-469 du 10 juillet 1989.

JORF no 0151 du 30 juin 2012 page 10673 texte nํ16 ARRETE Arrêté du 26 juin 2012 fixant les conditions d'exploitation des établissements chargés d'organiser les stages de sensibilisation à la sécurité routière NOR : INTS1226850A

Arrêté du 26 juin 1992 Article 223-5 du code de la route. Modifié par Décret no 2012-688 du 7 mai 2012 relatif à l'enseignement de la conduite et à l'animation de stages de sensibilisation à la sécurité routière. 


\section{NOTES}

1. Nous distinguerons le but recherché (la sécurité routière) de l'organisme (la Sécurité routière) par l'usage de la majuscule sur le substantif.

\section{RÉSUMÉS}

Lors d'un stage de récupération de points de permis de conduire, nous avons mené une recherche sur l'activité réelle mobilisée par les formateurs pendant les deux jours de stage en regard des prescriptions nationales arrêtées par la Sécurité routière. Ces stages étant assurés par des binômes, les co-animations plus ou moins imposées par les hasards d'affectations des stages, font cohabiter des conceptions sur l'apprentissage, sur la conduite routière, sur les relations avec la loi souvent différentes. À partir de l'hypothèse que les formateurs doivent concevoir la formation dans l'usage à partir des prescriptions nationales peu flexibles, nous avons procédé à un recueil de traces de l'activité des formateurs pendant le stage, ainsi qu'à des entretiens d'autoconfrontation simple. Nous mettons en évidence les genèses instrumentales opérées par les formateurs vis-à-vis de ce dispositif de formation considéré comme un artefact, leurs concepts pragmatiques en jeu, ainsi que sur les combinaisons de gestes professionnels d'ajustement qu'ils ont convoqués pour s'ajuster aux imprévus qu'ils ont rencontrés. Nos résultats mettent ainsi en lumière que les binômes doivent réaliser des conceptions alternées dans l'usage pour pouvoir assurer ces formations, préserver la paix sociale lors de ces stages et rester cohérents avec leurs valeurs, leurs visions du monde et leurs propres manières de se comporter sur la route.

During a training course to recover driving licence points in France, we led research to compare the actual activity mobilized by the trainers during the two-day course with national road safety rules. As these training sessions are given by teams of two persons who are more or less randomly allocated to the courses, they often bring together differing conceptions of learning, driving and relationships with the law. Working from the hypothesis that trainers have to continue the work of conception initially developed on the basis of relatively inflexible national rules, we traced trainer activity throughout the course and conducted self-confrontation interviews. We highlight the instrumental geneses operated by the trainers with regard to this training device, which is considered as an artefact, the pragmatic concepts at play, and the combinations of professional gestures of adjustment employed to cope with unexpected incidents. Our results show that the training team have to develop alternative content as they go, in order to be able to conduct the training, preserve social peace during the courses and remain coherent with their values, their visions of the world and their own ways of behaving on the road. 
INDEX

Mots-clés : sécurité routière, conceptions alternées dans l'usage, imprévus, gestes professionnels d'ajustement, activité

Keywords : road safety, alternative conception in the use, unexpected incidents, the professional gesture of adjustment, activity

\section{AUTEUR}

\section{ALAIN JEAN}

Faculté d'éducation ESPE, LIRDEF équipe TF\&D. Université de Montpellier, 2 place M Godechot, BP 4152, 34092 Montpellier Cedex 5

alain.jean@fde.univ-montp2.fr 